\title{
Interacting dark energy model and thermal stability
}

\author{
Pritikana Bhandari ${ }^{1, \mathrm{a}}$, Sourav Haldar ${ }^{1, \mathrm{~b}}$, Subenoy Chakraborty ${ }^{1, \mathrm{c}}$ \\ ${ }^{1}$ Department of Mathematics, Jadavpur University, Kolkata, West Bengal 700032, India
}

Received: 7 August 2017 / Accepted: 24 November 2017 / Published online: 7 December 2017

(C) The Author(s) 2017. This article is an open access publication

\begin{abstract}
In the background of the homogeneous and isotropic FLRW model, the thermodynamics of the interacting DE fluid is investigated in the present work. By studying the thermodynamical parameters, namely the heat capacities and the compressibilities, both thermal and mechanical stability are discussed and the restrictions on the equation of state parameter of the dark fluid are analyzed.
\end{abstract}

\section{Introduction}

The present era of cosmic evolution is a challenging issue to the cosmologists due to the recent observational predictions [1-9]. The explanation of the late-time accelerated expansion of the Universe has become one of the biggest and open problems in modern cosmology today. In the framework of Einstein gravity, the most reasonable description for this accelerating phase introduces some hypothetical exotic matter, known as dark energy (DE) (having large negative pressure), which comprises about $70 \%$ of the total energy density [10] of the Universe. The simplest as well as a large number of available observational data supported candidate for the DE is the cosmological constant $\Lambda$, associated with the zero point energy of the quantum fields. In spite of this great success of $\Lambda$-cosmology, it suffers from serious objections in the interface of cosmology and particle physics, namely the Cosmological Constant problem [11,12] and the Coincidence problem [13]. As a result of these serious problems in $\Lambda$, several alternative dynamical DE models have been proposed and studied for the last several years [14] but still the Cosmological Constant is the best DE candidate supporting the observational results. Furthermore, most of the proposed DE models try to adjust the data seamlessly, so observational data are not sufficient to decide between different kinds of

\footnotetext{
a e-mail: pritikanab@gmail.com

b e-mail: sourav.math.ju@gmail.com

c e-mail: schakraborty.math@gmail.com
}

DE. Hence, one has tried to invoke the Interacting DE model for a better understanding of the mechanism behind this cosmic acceleration.

In recent years, cosmological theories with interacting dark fluids are receiving much attention due to their ability to address the small value of the cosmological constant and to have a reasonable explanation to the cosmic coincidence problem [15-17]. Further, from observational point of view, recent data favor the late time interaction between DM and DE [18-23]. Also the coupling parameter in the interaction can be measured by various observations [21-30]. Moreover, it is speculated that class of interacting dark fluid models may resolve the current tensions on $\sigma_{s}$ and the local value of the Hubble constant $H_{0}$ [22-31]. Additionally, cosmologists are of the opinion that the interaction between the dark sectors may leave an imprint on the perturbation analysis and as a result there may be significant changes in the lowest multipoles of the CMB spectrum $[32,33]$. The motivation of the present paper is in this direction.

The thermodynamical study of DE is an important aspect for a better understanding of the unknown nature of DE. As the thermodynamical laws are applicable to all types of macroscopic systems and are based on experimental evidence, in contrast to classical mechanics or electromagnetism, thermodynamics does not predict specific numerical values for observables; rather it sets limit on physical processes. Hence the thermodynamic behavior of the cosmic fluid may give some clue to unveil the unknown nature of the content of the Universe (i.e. the hypothetical DE). In the recent past Barboza et al. [34] investigated the thermodynamic aspects of DE fluids. They analyzed both thermal and mechanical stability demanding the positivity of the heat capacities and compressibility of the dark fluid. They showed that due to the stability of the DE fluid, it should have negative constant equation of state parameter and it was also shown to be in contradiction with the observational constraints imposed by type Ia supernova, BAO and $H(z)$ data on a general DE fluid. Hence they concluded that DE 
fluid models are unphysical from a thermodynamical point of view. In the following sections, we shall apply the thermodynamical laws and the stability criteria for thermal equilibrium to impose bounds on the equation of state parameter [40] of the interacting DE so that the analysis may constrain or even rule out some DE models. The paper is organized as follows: Sect. 2 deals with cosmic evolution and thermodynamical analysis. The interrelation between different thermodynamical parameters and stability of the system have been presented in Sect. 3. Finally, in Sect. 4, there is a brief discussion and concluding remarks.

\section{Cosmic evolution and thermodynamic analysis}

In the background of the homogeneous and isotropic spatially flat FLRW model, the evolution of the Universe due to recent observations is considered in terms of a cosmic fluid in the form of interacting dark fluids, consisting of dark matter (DM) and dark energy (DE). So the Friedmann equations take the form

$3 H^{2}=\rho_{m}+\rho_{d}$

$2 \dot{H}+3 H^{2}=-p_{m}-p_{d}$,

with energy conservation equations

$\dot{\rho_{m}}+3 H\left(1+\omega_{m}\right) \rho_{m}=Q$,

$\dot{\rho_{d}}+3 H\left(1+\omega_{d}\right) \rho_{d}=-Q$,

where $\left(\rho_{m}, p_{m}\right)$ and $\left(\rho_{d}, p_{d}\right)$ are, respectively, the energy density and thermodynamic pressure of DM and DE, $\omega_{m}=$ $\frac{p_{m}}{\rho_{m}}, \omega_{d}=\frac{p_{d}}{\rho_{d}}$ are the equation of state parameters for the two dark fluids and the function $Q$ (with correct physical dimension) is the interaction between two fluids. The positive or negative sign of $\mathrm{Q}$ indicates an energy and/or momentum flow from DE to DM or vice versa. However, to alleviate the Coincidence problem and compatibility with the second law of thermodynamics, Q is normally chosen to be positive [17,35-37]. But recently, from an observational point of view, it has been shown that current observational data can favor the late time interaction in the dark sector with $Q<0[18,38]$. It is well known that the above two fluids can be combined as a single fluid having energy density $\rho_{T}=\rho_{m}+\rho_{d}, p_{T}=p_{m}+p_{d}$ is the pressure of the combined fluid with $\omega_{T}=\left(\omega_{m} \Omega_{m}+\omega_{d} \Omega_{d}\right)$ the effective equation of state of the single hypothetical fluid. Here $\Omega_{m}$ and $\Omega_{d}$ are the density parameters of the two dark sectors. However, in the present problem we choose $\omega_{m}=0$ i.e. the cold dark matter $\left(\omega_{m} \neq 0\right.$ corresponds to hot dark matter) because for hot DM (a non-cold relic) one must take the complete set of Boltzmann equations which has not been considered in the following thermodynamical analysis. As a result we have $\omega_{T}=\omega_{d} \Omega_{d}$ and $p_{T}=p_{d}$. Thus the above Friedmann equations and the conservation equations can effectively be written

$3 H^{2}=\rho_{T}, \quad 2 \dot{H}+3 H^{2}=-\left(\rho_{T}+p_{T}\right)$

and

$\dot{\rho}_{T}+3 H\left(1+\omega_{T}\right) \rho_{T}=0$.

Now, from the point of view of thermodynamical analysis, the internal energy of the cosmic system is defined by

$I_{E}=\rho_{T} c^{2} V$

where $\rho_{T}$ is the usual density of the effective fluid and $V=a^{3}(t) V_{0}$ is the physical volume of the Universe at a given time. Here the suffix ' 0 ' indicates the value of the corresponding variable at present time with the convention $a_{0}=1$ and $a(t)$ is called the scale factor of the Universe as all physical distance scales with the same factor ' $a$ ' due to the homogeneous and isotropic nature of the model. From the thermodynamic point of view, if the expansion of the Universe is assumed to be reversible and adiabatic in nature then the first law of thermodynamics (an energy conservation equation) takes the form

$T d S=d I_{E}+p_{T} d V$

and consequently one gets the fluid's conservation law $\left(T_{\nu ; \mu}^{\mu}=0\right)$ :

$d \ln \rho_{T}+\left(1+\omega_{T}\right) d \ln V=0$.

Choosing temperature $(T)$ and volume $(V)$ as the independent thermodynamical variables i.e. $\rho_{T}=\rho_{T}(T, V)$, for $d S$ to be an exact differential (from (8)) gives [34,39]

$d \ln T=-\omega_{T} d \ln V+d \ln \left|1+\omega_{T}\right|$

or equivalently combining with Eq. (9) one can integrate Eq. (10) to give

$\left(1+\omega_{T}\right) \frac{\rho_{T} V}{T}=\left(1+\omega_{0}\right) \frac{\rho_{0} V_{0}}{T_{0}}=$ constant

i.e.

$I_{E}=I_{0}\left(\frac{1+\omega_{0}}{1+\omega_{T}}\right) \frac{T}{T_{0}}$

Here Eq. (11) can be considered as the modified ideal gas law for a fluid having a variable equation of state. As for any terrestrial fluid, it is possible to have an experimental determination of the thermodynamical derivatives, namely the heat 
capacity, the compressibility and the thermal expandability; so it will be interesting to analyze these variables in the present context. In this context, it should be mentioned that these derived thermodynamical variables, namely the heat capacities and the compressibilities of the fluid, are respectively related to the thermal and mechanical stability of the system.

Formally, in classical thermodynamics, the heat capacity of a fluid is defined as the ratio of an absorbed heat $(d Q=$ $T d S)$ due to an increase in the fluid's temperature $(d T)$. As the fluid can be heated either at constant volume or at constant pressure, one gets two types of heat capacities, which can be formally defined using the first law of thermodynamics (i.e. Eq. (8)): [41]

$$
\begin{aligned}
C_{v} & =\left(\frac{\partial I_{E}}{\partial T}\right)_{V}, \\
C_{p} & =\left(\frac{\partial h}{\partial T}\right)_{p} .
\end{aligned}
$$

They are termed the heat capacity at constant volume and the heat capacity at constant pressure, respectively. Also,

$h=I_{E}+p V$

is known as the enthalpy of the fluid.

Now using the expression for the internal energy from Eq. (12) and the integrability condition (10) one gets

$C_{p}=\left(1+\omega_{T}\right) \frac{I_{E}}{T}=\left(1+\omega_{0}\right) \frac{I_{0}}{T_{0}}=\mathrm{constant}$

and

$C_{v}=\frac{d \ln V}{\left\{\left(1+\omega_{T}\right) d \ln V-d \ln \omega_{T}\right\}} C_{p}$.

Thus the specific heat (the heat capacity per unit mass) at constant pressure is

$c_{p}=\frac{C_{p}}{\rho_{0} V_{0}}=\frac{c^{2}\left(1+\omega_{0}\right)}{T_{0}}$.

It is well known that the temperature for the relativistic matter is $T_{r}=2.725 \mathrm{~K}$, so the ratio of the specific heats at this era will be $\sim 10^{13} \mathrm{cal} \mathrm{g}^{-1} \mathrm{~K}^{-1}$. As the temperatures of the other matter components are smaller than that for relativistic matter, the specific heat for the relativistic matter can be taken as the lower limit for the Universe's specific heat. Hence the Universe can be imagined as a huge thermal reservoir. However, technologically we are at present not in a position to isolate a cosmologically significant part of the Universe, where, by providing an enormous amount of heat, it is possible to measure the temperature change and hence the specific heat of the portion of the Universe.

\section{Thermal interrelation and stability of the system}

Assuming temperature and pressure as the independent thermodynamic variables the variation in volume can be expressed as

$d V=V\left(\alpha d T-\kappa_{T} d p\right)$

where

$\alpha=\frac{1}{V}\left(\frac{\partial V}{\partial T}\right)_{p}$

is known as the thermal expansivity, while the isothermal compressibility $\left(\kappa_{T}\right)$ gives a measure of the relative change of volume with increasing pressure at fixed temperature i.e.

$\kappa_{T}=-\frac{1}{V}\left(\frac{\partial V}{\partial p}\right)_{T}$.

Similarly, in adiabatic situation compressibility $\kappa_{S}$ is defined keeping the entropy fixed:

$\kappa_{S}=-\frac{1}{V}\left(\frac{\partial V}{\partial p}\right)_{S}$.

In an isothermal thermodynamic scenario the compressibility and expansibility are related by [34]

$\frac{\alpha}{\kappa_{T}}=\left(\frac{\partial p}{\partial T}\right)_{V}$.

The well-known relation between heat capacities and compressibilities in the adiabatic and isothermal thermodynamical scenarios is given by [42]

$\frac{\kappa_{s}}{\kappa_{T}}=\frac{C_{v}}{C_{p}}$.

From the very definition (20) using Eq. (16) and the integrability condition (10), the thermal expansivity has the explicit expression

$$
\begin{aligned}
\alpha= & \frac{C_{p}}{\left(1+\omega_{T}\right) \rho_{T} V} \\
& \times\left[1+\frac{d \omega_{T}}{\omega_{T}\left\{d \omega_{T}-\omega_{T}\left(1+\omega_{T}\right) d \ln V\right\}}\right] .
\end{aligned}
$$

Hence, Eq. (23) gives

$\kappa_{T}=\frac{\alpha V}{C_{p}}$

and the thermodynamical identity (24) shows that

$\kappa_{s}=\frac{\alpha V C_{V}}{C_{p}^{2}}$. 
Table 1 Restrictions for stability of the system

\begin{tabular}{ll}
\hline $\begin{array}{l}\text { Restriction on the } \\
\text { equation of state } \\
\text { parameter } \omega_{T}\end{array}$ & Condition for stability \\
\hline$\omega_{T}>0$ & $V \frac{d \omega_{T}}{d V}<\omega_{T}^{2}$ or $V \frac{d \omega_{T}}{d V}>\omega_{T}\left(1+\omega_{T}\right)$ \\
$-1<\omega_{T}<0$ & $\omega_{T}\left(1+\omega_{T}\right)<V \frac{d \omega_{T}}{d V}<\omega_{T}^{2}$ \\
$\omega_{T}<-1$ & Unstable \\
\hline
\end{tabular}

From Eq. (17), the compressibilities are related by

$\kappa_{s}=\frac{d \ln V}{\left\{\left(1+\omega_{T}\right) d \ln V-d \ln \omega_{T}\right\}} \kappa_{T}$.

We shall now discuss the thermal stability of the present system assuming work done only due to the volume variation of the thermal system. It is well known that for stable equilibrium the second order variation of the internal energy [34,42], i.e.

$\delta^{2} I_{E}=\delta T \delta S-\delta p \delta V$,

should be positive semi-definite i.e. $\delta^{2} I_{E} \geq 0$. Now choosing $(T, V)$ or $(S, p)$ as the independent thermodynamical variables the above second order variation can be written as

$$
\delta^{2} I_{E}=\begin{gathered}
\frac{C_{v}}{T} \delta T^{2}+\frac{1}{V \kappa_{T}} \delta V^{2} \\
\text { or } \\
\frac{T}{C_{p}} \delta S^{2}+V \kappa_{s} \delta p^{2} .
\end{gathered}
$$

Hence for stability of the system the thermodynamical parameters, namely $\left(C_{p}, C_{v}, \kappa_{T}, \kappa_{s}\right)$, should be non-negative, i.e.

$C_{v}, C_{p}, \kappa_{T}, \kappa_{s} \geq 0$.

From Eq. (16) it is evident that $C_{p}$ is constant and positive definite.

The non-negativity of the other derived thermodynamical parameters is examined from the above interrelations and the results are presented in Table 1.

In particular, for the cold DM under consideration we have $\omega_{T}=\omega_{d} \Omega_{d}$, so the above stability conditions can explicitly be written in terms of the equation of state parameter $\omega_{d}$ for the DE fluid; see Table 2.

The feasible regions of $\omega_{d}$ for the stability criteria presented in Table 2 are shown graphically in Figs. 1 and 2 for the first two rows of the table.

Due to the restrictions on the equation of state parameter for the stability criteria, it is natural to examine the constraint on the interaction $Q$ between the dark fluids. As for the stability condition, $\left(1+\omega_{d}\right)$ is always positive (i.e. the DE fluid is non-phantom in nature) so from the conservation equation (4) we must have
Table 2 Restrictions on DE equations of state for stability criteria

\begin{tabular}{ll}
\hline $\begin{array}{l}\text { Restriction on the } \\
\text { equation of state } \\
\text { parameter } \omega_{d}\end{array}$ & Condition for stability \\
\hline$\omega_{d}>0$ & $\frac{V}{\Omega_{d}^{2}} \frac{d\left(\omega_{d} \Omega_{d}\right)}{d V}<\omega_{d}^{2}$ or $\frac{V}{\Omega_{d}} \frac{d\left(\omega_{d} \Omega_{d}\right)}{d V}>$ \\
& $\omega_{d}\left(1+\omega_{d} \Omega_{d}\right)$ or equivalently (assuming \\
& $\Omega_{d}=$ constant) $\frac{a^{3}}{1-\Omega_{d} a^{3}}<\omega_{d}<\frac{1}{\ln a^{-3 \Omega_{d}}}$ \\
$-1<\omega_{d}<0$ & $\omega_{d}\left(\frac{1}{\Omega_{d}}+\omega_{d}\right)<\frac{V}{\Omega_{d}^{2}} \frac{d \omega_{d}}{d V}<\omega_{d}^{2}$ or \\
& equivalently $\frac{-\frac{1}{\Omega_{d}} a^{3 \Omega_{d}}}{1+a^{3 \Omega_{d}}}<\omega_{d}<0$ \\
$\omega_{d}<-1$ & Unstable
\end{tabular}

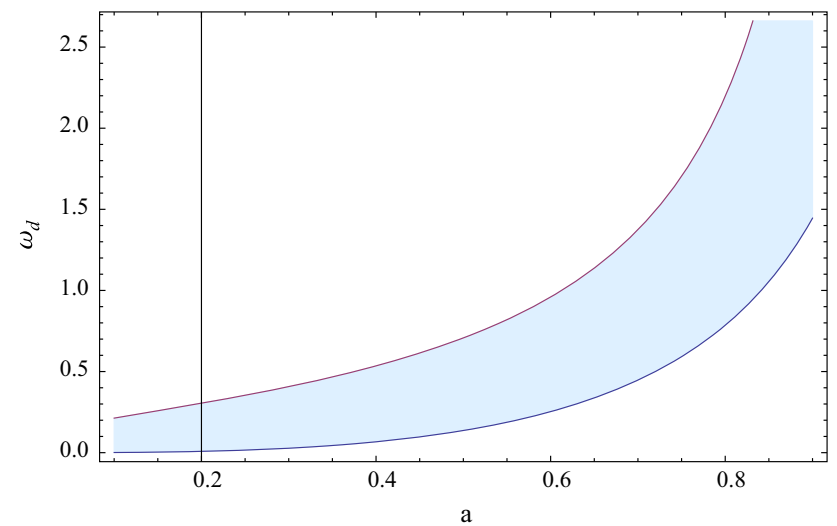

Fig. 1 The shaded region shows the valid region for $\omega_{d}(>0)$ under stability criteria for $\Omega_{d}=0.68$

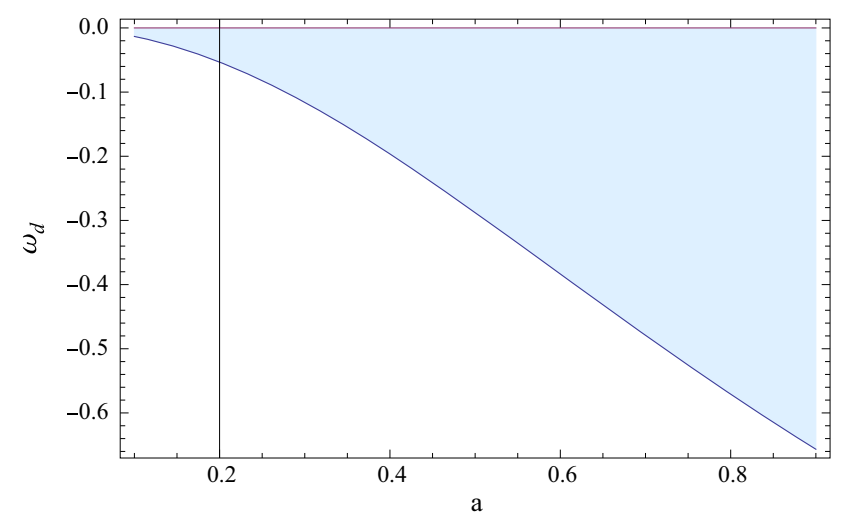

Fig. 2 The shaded region shows the valid region for $\omega_{d}\left(-1<\omega_{d}<\right.$ 0 ) under stability criteria for $\Omega_{d}=0.68$

$Q<\left|\dot{\rho_{d}}\right|$

In particular, if the interaction is taken as $Q=3 \epsilon H \rho_{m}$ ( $\epsilon$ is a small coupling parameter) then solving the energy conservation equations the above restriction on $Q$ has the explicit form (with $\rho_{m_{0}}$ and $\rho_{0}$ are integration constants) 


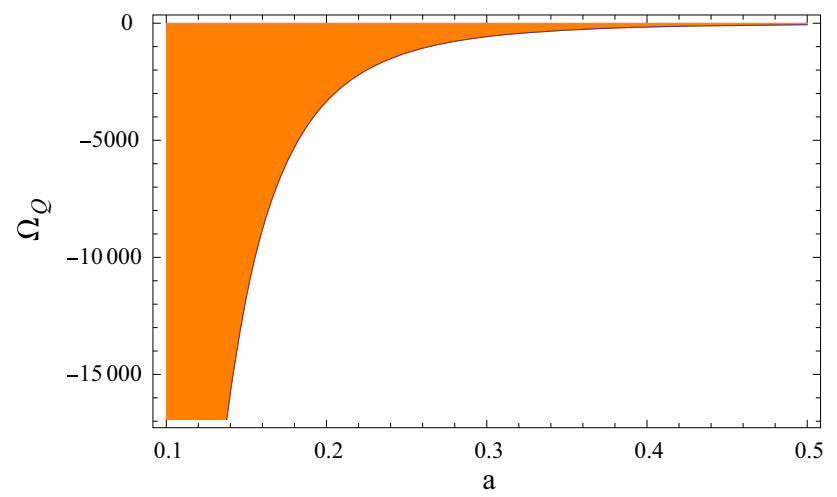

Fig. 3 The shaded portion is the admissible domain for $\Omega_{Q}$ in the negative region for $\omega_{d}=0.45, \Omega_{m_{0}}=0.30$ and $\epsilon=0.01$

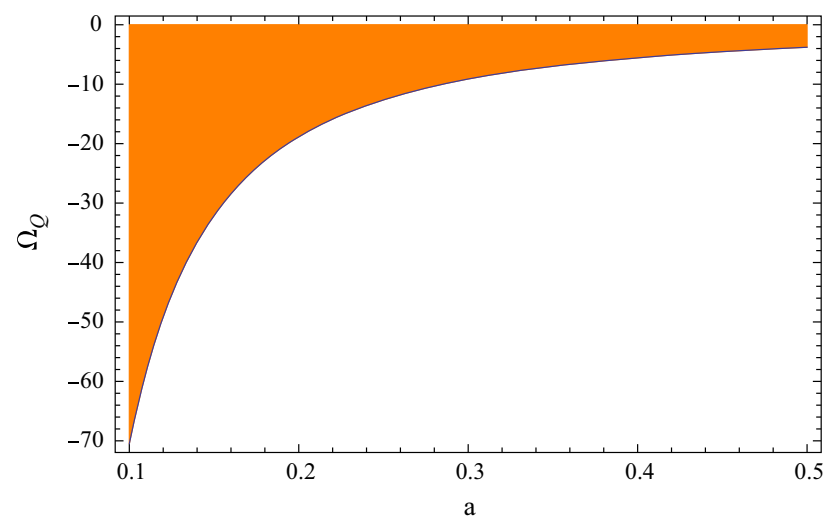

Fig. 4 The shaded portion is the admissible domain for $\Omega_{Q}$ in the negative region for $\omega_{d}=-0.45, \Omega_{m_{0}}=0.30$ and $\epsilon=0.01$

$Q<3 H a^{-3(1-\epsilon)}\left|\frac{\epsilon(1-\epsilon) \rho_{m_{0}}}{\left(\epsilon+\omega_{d}\right)}-\left(1+\omega_{d}\right) \rho_{0} a^{-3\left(\epsilon+\omega_{d}\right)}\right|$

or equivalently in terms of the density parameter

$$
\begin{aligned}
\Omega_{Q}< & 3 a^{-3(1-\epsilon)} \\
& \times\left|\frac{\epsilon(1-\epsilon) \Omega_{m_{0}}}{\left(\epsilon+\omega_{d}\right)}-\left(1+\omega_{d}\right)\left(1-\Omega_{m_{0}}\right) \rho_{0} a^{-3\left(\epsilon+\omega_{d}\right)}\right|,
\end{aligned}
$$

with $\Omega_{Q}=\frac{8 \pi G}{3 H_{0}^{2}} \cdot \frac{Q}{H}$, and $\Omega_{m_{0}}=\frac{\rho_{m_{0}}}{\left(\frac{3 H_{0}^{2}}{8 \pi G}\right)}$.

Now, the accessible regions of $\Omega_{Q}$ (i.e. $Q$ ) for two different choices of the parameters involved are shown in Figs. 3 and 4.

\section{Brief discussion and concluding remarks}

The present work is an attempt to address the present challenging question: "What is the cause of the present accelerated expansion of the Universe?". So far there is no definite answer. One possible cause for this accelerated expansion is the presence of the unknown matter component, while some people believe that Einstein's general relativity does not work at large scale. For the DE option, the simplest choice is the cosmological constant, but this shows some severe problems. Therefore, several models have been proposed for dynamical DE. The present work is a small attempt to examine the DE possibility from the thermodynamical point of view. We have studied the thermodynamical aspects of the cosmic fluid in the form of interacting DE in the background of an expanding, homogeneous and isotropic FRW model of the Universe. Although the interaction term $Q$ should be taken to be positive from the thermodynamical point of view, recent observations suggest it may be negative also. An estimation of the specific heat of the Universe has been made and the constraints imposed by classical thermodynamics on the cosmic fluid have been examined. As it is desirable that the cosmic fluid should reach the thermodynamic stability, such a requirement is shown in tabular form. It is found that the phantom nature of the dark energy fluid is not thermodynamically stable. Also we have seen from the analysis that $\omega_{T}=-1$ is unphysical i.e. the vacuum energy as DE cannot be invoked as it is physically different from a DE fluid. However, our analysis shows that the DE equation of state parameter $\omega_{d}$ in the quintessence era (i.e. $-1<\omega_{d}<-\frac{1}{3}$ ) is thermodynamically stable (with some restrictions). The possible ranges of $\omega_{d}$ are shown in Table 2 and their graphical representations are presented in Figs. 1 and 2. Also, the admissible regions of $Q$ for specific choices of the parameters involved are shown diagrammatically in Figs. 3 and 4. It is found that both signs of $Q$ are possible for thermal stability. So $Q$ supports the recent observations. Therefore, we may conclude that interacting dark fluids with effective equation of state parameter $\omega_{d}>-1$ are thermodynamically stable and may be a possible answer to the question of the unknown DE matter.

Acknowledgements The author PB acknowledges DST-INSPIRE and SH acknowledges UGC-JRF for awarding Research fellowship. The author SC is thankful to the Inter-University Centre for Astronomy and Astrophysics (IUCAA), Pune, India for research facilities at Library. SC also acknowledges the UGC-DRS Programme in the Department of Mathematics, Jadavpur University.

Open Access This article is distributed under the terms of the Creative Commons Attribution 4.0 International License (http://creativecomm ons.org/licenses/by/4.0/), which permits unrestricted use, distribution, and reproduction in any medium, provided you give appropriate credit to the original author(s) and the source, provide a link to the Creative Commons license, and indicate if changes were made.

Funded by $\mathrm{SCOAP}^{3}$. 


\section{References}

1. S. Perlmutter et al., [Supernova Cosmology Project Collaboration], Measurements of Omega and Lambda from 42 high redshift supernovae. Astrophys. J. 517, 565 (1999). arXiv:astro-ph/9812133

2. A.G. Riess et al., [Supernova Search Team], Observational evidence from supernovae for an accelerating universe and a cosmological constant. Astron. J. 116, 1009 (1998). arXiv:astro-ph/9805201

3. P. de Bernardis et al., [Boomerang Collaboration], A flat universe from high resolution maps of the cosmic microwave background radiation. Nature 404, 955 (2000). arXiv:astro-ph/0004404

4. W.J. Percival et al., [2dFGRS Collaboration], The 2dF Galaxy Redshift Survey: the power spectrum and the matter content of the universe. Mon. Not. R. Astron. Soc. 327, 1297 (2001). arXiv:astro-ph/0105252

5. D.N. Spergel et al., [WMAP Collaboration], First year Wilkinson Microwave Anisotropy Probe (WMAP) observations: determination of cosmological parameters. Astrophys. J. Suppl. 148, 175 (2003). arXiv:astro-ph/0302209

6. B. Jain, A. Taylor, Cross-correlation tomography: measuring dark energy evolution with weak lensing. Phys. Rev. Lett. 91, 141302 (2003). arXiv:astro-ph/0306046

7. M. Tegmark et al., [SDSS Collaboration], Cosmological parameters from SDSS and WMAP. Phys. Rev. D 69, 103501 (2004). arXiv:astro-ph/0310723

8. D.J. Eisenstein et al., [SDSS Collaboration], Detection of the baryon acoustic peak in the large-scale correlation function of SDSS luminous red galaxies. Astrophys. J. 633, 560 (2005). arXiv:astro-ph/0501171

9. E. Komatsu et al., [WMAP Collaboration], Seven-year Wilkinson microwave anisotropy probe (WMAP) observations: cosmological interpretation. Astrophys. J. Suppl. 192, 18 (2011). arXiv: 1001.4538 [astro-ph.CO]

10. P.A.R. Ade et al., [Planck Collaboration], Planck 2015 results. XIII. Cosmological parameters. Astron. Astrophys. 594, A13 (2016). arXiv: 1502.01589 [astro-ph.CO]

11. S. Weinberg, The cosmological constant problem. Rev. Mod. Phys. 61, 1 (1989)

12. T. Padmanabhan, Cosmological constant: the weight of the vacuum. Phys. Rep. 380, 235 (2003). arXiv:hep-th/0212290

13. P.J. Steinhardt, A quintessential introduction to dark energy. Philos. Trans. R. Soc. Lond. A 361, 2497 (2003)

14. L. Amendola, S. Tsujikawa, Dark Energy: Theory and Observations (Cambridge University Press, Cambridge, 2010)

15. Y .L. Bolotin, A. Kostenko, O .A. Lemets, D .A. Yerokhin, Cosmological evolution with interaction between dark energy and dark matter. Int. J. Mod. Phys. D 24(03), 1530007 (2014). arXiv:1310.0085 [astro-ph.CO]

16. B. Wang, E. Abdalla, F. Atrio-Barandela, D. Pavon, Dark matter and dark energy interactions: theoretical challenges, cosmological implications and observational signatures. Rept. Prog. Phys. 79(9), 096901 (2016). arXiv:1603.08299 [astro-ph.CO]

17. G.S. Sharov, S. Bhattacharya, S. Pan, R .C. Nunes, S. Chakraborty, A new interacting two fluid model and its consequences. Mon. Not. R. Astron. Soc. 466(3), 3497 (2017). arXiv:1701.00780 [gr-qc]

18. V. Salvatelli, N. Said, M. Bruni, A. Melchiorri, D. Wands, Indications of a late-time interaction in the dark sector. Phys. Rev. Lett. 113(18), 181301 (2014). arXiv:1406.7297 [astro-ph.CO]

19. J. Sola, A. Gomez-Valent, J. de Cruz Prez, Hints of dynamical vacuum energy in the expanding Universe. Astrophys. J. 811, L14 (2015). arXiv:1506.05793 [gr-qc]

20. J. Sola, J. de Cruz Prez, A. Gomez-Valent, R.C. Nunes, Dynamical vacuum against a rigid cosmological constant. arXiv:1606.00450 [gr-qc]
21. R .C. Nunes, S. Pan, E .N. Saridakis, New constraints on interacting dark energy from cosmic chronometers. Phys. Rev. D 94(2), 023508 (2016). arXiv:1605.01712 [astro-ph.CO]

22. S. Kumar, R.C. Nunes, Probing the interaction between dark matter and dark energy in the presence of massive neutrinos. Phys. Rev. D 94(12), 123511 (2016). arXiv:1608.02454 [astro-ph.CO]

23. C. van de Bruck, J. Mifsud, J. Morrice, Testing coupled dark energy models with their cosmological background evolution. Phys. Rev. D 95(4), 043513 (2017). arXiv:1609.09855 [astro-ph.CO]

24. T. Yang, Z .K. Guo, R .G. Cai, Reconstructing the interaction between dark energy and dark matter using Gaussian Processes. Phys. Rev. D 91(12), 123533 (2015). arXiv:1505.04443 [astroph.CO]

25. W. Yang, L. Xu, Cosmological constraints on interacting dark energy with redshift-space distortion after Planck data. Phys. Rev. D 89(8), 083517 (2014). arXiv:1401.1286 [astro-ph.CO]

26. W. Yang, L. Xu, Coupled dark energy with perturbed Hubble expansion rate. Phys. Rev. D 90(8), 083532 (2014). arXiv:1409.5533 [astro-ph.CO]

27. W. Yang, H. Li, Y. Wu, J. Lu, Cosmological constraints on coupled dark energy. JCAP 1610(10), 007 (2016). arXiv:1608.07039 [astroph.CO]

28. D .M. Xia, S. Wang, Constraining interacting dark energy models with latest cosmological observations. Mon. Not. R. Astron. Soc. 463(1), 952 (2016). arXiv: 1608.04545 [astro-ph.CO]

29. C. Caprini, N. Tamanini, Constraining early and interacting dark energy with gravitational wave standard sirens: the potential of the eLISA mission. JCAP 1610(10), 006 (2016). arXiv:1607.08755 [astro-ph.CO]

30. R. Murgia, S. Gariazzo, N. Fornengo, Constraints on the coupling between dark energy and dark matter from CMB data. JCAP 1604(04), 014 (2016). arXiv:1602.01765 [astro-ph.CO]

31. A. Pourtsidou, T. Tram, Reconciling CMB and structure growth measurements with dark energy interactions. Phys. Rev. D 94(4), 043518 (2016). arXiv:1604.04222 [astro-ph.CO]

32. W. Zimdahl, Interacting dark energy and cosmological equations of state. Int. J. Mod. Phys. D 14, 2319 (2005). arXiv:gr-qc/0505056

33. B. Wang, J. Zang, C.Y. Lin, E. Abdalla, S. Micheletti, Interacting dark energy and dark matter: observational constraints from cosmological parameters. Nucl. Phys. B 778, 69 (2007). arXiv:astro-ph/0607126

34. E.M. Barboza, R.C. Nunes, E.M.C. Abreu, J.A. Neto, Thermodynamic aspects of dark energy fluids. Phys. Rev. D 92(8), 083526 (2015)

35. D. Pavon, B. Wang, Le Chatelier-Braun principle in cosmological physics. Gen. Relativ. Gravity 41, 1 (2009). arXiv:0712.0565 [grqc]

36. S.K. Biswas, S. Chakraborty, Interacting dark energy in $f(T)$ cosmology: a dynamical system analysis. Int. J. Mod. Phys. D 24(07), 1550046 (2015). arXiv:1504.02431 [gr-qc]

37. N. Mahata, S. Chakraborty, Dynamical system analysis for DBI dark energy interacting with dark matter. Mod. Phys. Lett. A 30(02), 1550009 (2015). arXiv:1501.04441 [gr-qc]

38. S. Kumar, R.C. Nunes, Echo for interaction in the dark sector. arXiv: 1702.02143 [astro-ph.CO]

39. S. Weinberg, Entropy generation and the survival of protogalaxies in an expanding universe. Astrophys. J. 168, 175 (1971)

40. J.A.S. Lima, J.S. Alcaniz, Thermodynamics and spectral distribution of dark energy. Phys. Lett. B 600, 191 (2004). arXiv:astro-ph/0402265

41. H.B. Callen, Thermodynamics and an Introduction to Thermostatistics, 2nd edn. (Wiley, New York, 1985)

42. R. Kubo, Thermodynamics: An Advanced Course with Problems and Solutions (North-Holland, Amsterdam, 1968) 